\title{
In silico assessment of a computerised model-based glycaemic control approach in a Belgian medical intensive care unit
}

\section{S. Penning, B. Lambermont, T. Desaive, C. Pretty and J.G. Chase}

Problem:

Critical illness

Stress-induced hyperglycaemia

Reduced insulin sensitivity

Increased mortality

Glycaemic variability

Solution: $\quad$ Model-based glycaemic control: exogenous insulin/nutrition inputs

Dosing ? Timing ?

\section{(1) STAR framework}

New blood glucose

(BG) measurement

Blood glucose (BG)

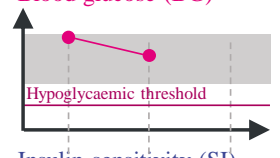

Insulin sensitivity (SI)

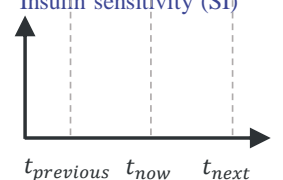

$t_{\text {previous }} t_{\text {now }} t_{\text {next }}$

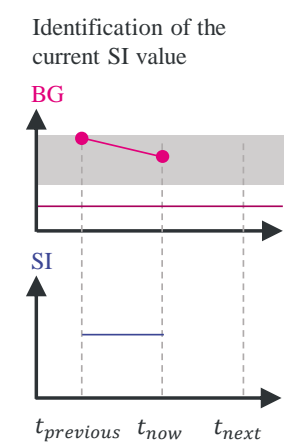

Insulin-only, target-to-range, patient-specific and adaptive glycaemic control approach
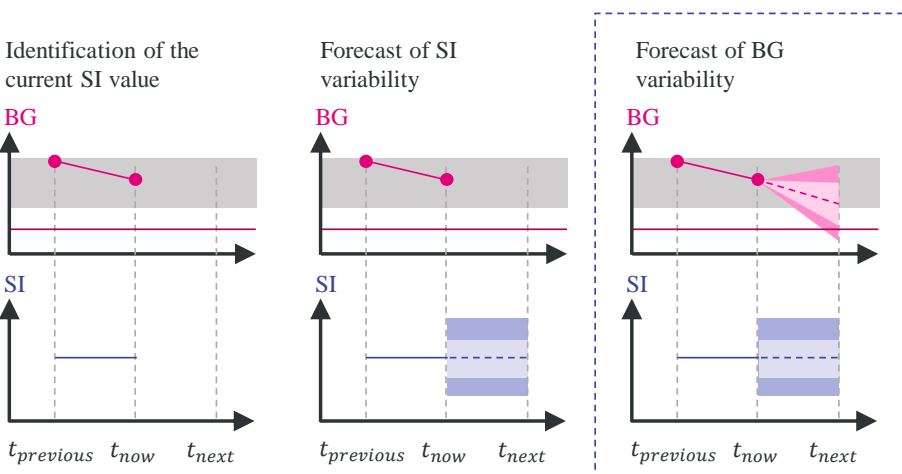

Hypoglycaemic risk assessment
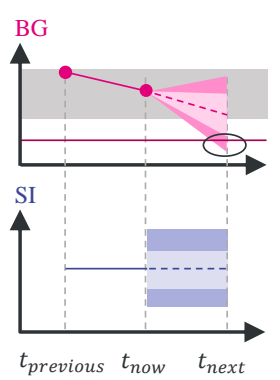

Frequency: 1, 2 or 3 hours Insulin rate: $0.0,1.0,1.5,2.0,2.5 \ldots 6.0 \mathrm{U} / \mathrm{h}$

\section{(2) Virtual patients}

Number of patients

$\%$ of males

Age (years)

SAPS II score

Initial glycaemia (mmol/L)

\section{0}

45.0

$68.0[54.0-76.0]$

$67.0[51.0-76.0]$

8.5 [7.3 - 9.9]
Identification of insulin sensitivity (SI) profile

Clinical data (glycaemic levels, insulin and nutrition rates/timing)

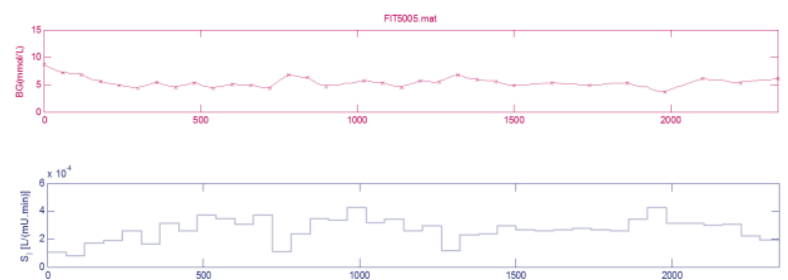

\section{(3) Virtual trials}

Virtual patient

Glycaemic outcome

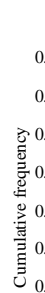

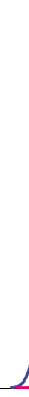

BG (mmol/L) Clinical protocol STAR-Liege 3
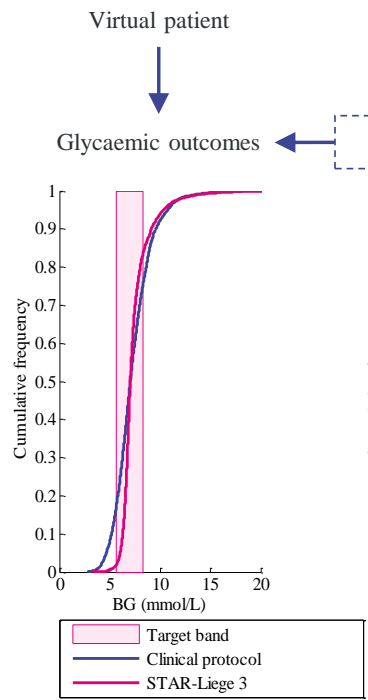

In silico assessment of the efficiency, safety and performance of the STAR framework

Virtual patient (hourly SI values)
Optimal insulin dosing and timing

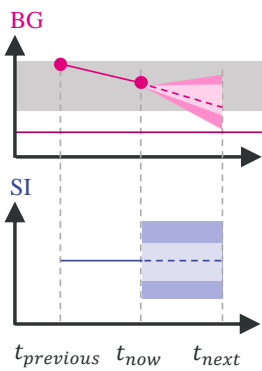

STAR framework
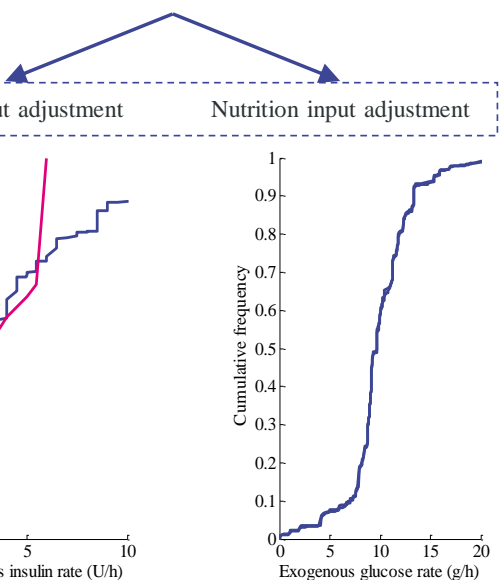

Total hours

Number of measurements

Blood glucose (BG) levels ( $\mathrm{mmol} / \mathrm{L})$

$\% \mathrm{BG} \geq 10.0 \mathrm{mmol} / \mathrm{L}$

$\%$ BG within 8.3-10.0 mmol/L

\% BG within 5.6-8.3 mmol/L

$\%$ BG within 4.4-5.6 mmol/L

$\% \mathrm{BG}<4.4 \mathrm{mmol} / \mathrm{L}$

$\% \mathrm{BG}<4.0 \mathrm{mmol} / \mathrm{L}$

$\% \mathrm{BG}<2.2 \mathrm{mmol} / \mathrm{L}$

Number of patients with $B G<2.2 \mathrm{mmol} / \mathrm{L}$

Exogenous insulin rate (U/h)

Exogenous glucose rate $(\mathrm{g} / \mathrm{h})$
Clinical protocol STAR-Liege 3

$\begin{array}{ll}5009 & 5014 \\ 2125 & 1912\end{array}$

$7.0[6.1-8.3]$

$7.0[6.7-7.7]$

$\begin{array}{ll}7.76 & 6.12\end{array}$

17.04

10.47

58.98

81.82

$13.12 \quad 1.49$

3.10

0.10

1.23

0.04

0.00

0.00

0

0

$3.0[2.0-6.5] \quad 3.5[2.0-6.0]$

$9.7[8.8-11.7] \quad 9.7[8.8-11.6]$

Reduction of clinical workload Safe, effective glycaemic control

STAR = big improvement over the current clinical protocol 